\title{
DECOMPOSITION OF GRADED MODULES
}

\author{
CARY WEBB ${ }^{1}$
}

\begin{abstract}
In this paper, the primary objective is to obtain decomposition theorems for graded modules over the polynomial ring $k[x]$, where $k$ denotes a field. There is some overlap with recent work of Höppner and Lenzing. The results obtained include identification of the free, projective, and injective modules. It is proved that a module that is either reduced and locally finite or bounded below is a direct sum of cyclic submodules. Pure submodules are direct summands if they are bounded below. In such case, the pure submodule is itself a direct sum of cyclic submodules. It is also noted that Cohen and Gluck's Stacked Bases Theorem remains true if the modules are graded.
\end{abstract}

I. Introduction. The purpose of this study is to obtain decomposition theorems for graded $k[x]$-modules, where $k$ denotes a field. Such a module is a direct sum, in the upgraded sense, of $k$-vector spaces $V_{i}$ indexed over the positive and negative integers. The operation $x$ on $V_{i}$ is an (arbitrary) linear transformation $x_{i}: V_{i} \rightarrow V_{i+1}$. (Thus $x$ actually represents a countable family of distinct linear transformations.) The $V_{i}$ 's are called the homogeneous components of $V$.

Our most important kind of results are those which decompose a graded $k[x]$ module into a direct sum of cyclic submodules and also those results which show how a submodule can be a direct summand. Important too but more easily derived are the identities of injective objects and the fact that all projective objects are free.

Graded $k[x]$-modules can be considered a special case in the theory of quivers over a ring. A quiver is a directed graph, that is, a set (of vertices) together with a set of arrows connecting (some of) the pairs of vertices. A graded $k[x]$-module is a representation of the quiver $\mathbf{Z}$, the ring of integers with the edges $n \rightarrow n+1$, over the field $k$. The general theory considers representations of arbitrary directed sets over arbitrary rings.

An early use of the idea of a quiver in representation theory is contained in [6]. Early appearances of quivers concern problems with finite dimensional algebras. In this regard, the references $[\mathbf{4}, \mathbf{5}, \mathbf{7}]$ are also relevant.

Morphisms between graded $k[x]$-modules $V$ and $V^{\prime}$ are of degree zero, sending $V_{i}$ into $V_{i}^{\prime}$. The graded nature of the morphisms implicitly defines which subsets are submodules (i.e., those generated by homogeneous elements). The same sort of comment can be made about direct summands, projectives, injectives, and free

Received by the editors January 30, 1982 and, in revised form, December 9, 1983 and August $15,1984$.

1980 Mathematics Subject Classification. Primary 13C06; Secondary 13F20, $20 \mathrm{~K} 25$.

Key words and phrases. Graded module, direct sum, pure submodule.

${ }^{1}$ This project was suggested by Irving Kaplansky who also made numerous helpful suggestions. Useful comments by an anonymous referee are also gratefully acknowledged. The author was supported in part by the NSF, grant SER 76-08610. Certain of the results presented here were also presented at the AMS meeting in St. Louis, April 11-12, 1975. 
graded $k[x]$-modules. The category of graded $k[x]$-modules is readily seen to be abelian.

It is natural to think of the origin of graded $k[x]$-modules to be in abelian groups (or, slightly more generally, modules over a PID). In this regard, the presentation in [9] is particularly relevant. In fact, it is true that the graded versions of the lemmas and theorems on pp. 14-25 of [9] carry over to graded $k[x]$-modules with only a straightforward change in terminology in both the statements of the results and their proofs.

There is some overlap between the approach and the results reported here and the work of Höppner and Lenzing [8]. The overlap includes the identification of projective and injective objects (our Propositions 1 and 2), the existence of rank 1 direct summands (our Propositions 2, 3 and 4 and Theorem 2), and the identity of countably generated torsion-free reduced graded $k[x]$-modules (our Theorem 2).

The work presented here was done during 1974-77. A partial announcement was made in $[\mathbf{1 1}]$.

It is possible that morphisms should be allowed to be of any degree and not merely degree zero. This would have only a small effect on the results we present here. For instance, all rank 1 free modules would be isomorphic if the degree were not constrained to be zero (i.e., a free module with a homogeneous generator of degree $n$ and one with a homogeneous generator of degree $m$ would be isomorphic by a morphism of degree $|m-n|)$.

II. Frees, projectives, and injectives. Free graded $k[x]$-modules have homogeneous bases. The normal things are true except, as was mentioned in the preceding paragraph, a set of $k[x]$ 's can be pasted together in different ways (specified by the number of 1's in the various components). Hence two free graded modules of the same rank need not be isomorphic by a graded isomorphism. It will be clear that the same sort of comment applies to injective graded modules.

Projective graded modules are necessarily free. This is shown in [3]. We record this fact in the following proposition.

Proposition 1. Projective graded $k[x]$-modules are free.

It can be shown by a common argument (which depends on Zorn's Lemma) that the injective objects are exactly those that are $x$-divisible. We represent by $P_{m}$ a copy of the injective $k\left[x^{-1}\right]$, where we mean $x \cdot 1=0$ and 1 is assigned degree $m$. We write $P$ if $m$ is of no consequence. $Q$ will denote the injective $k\left[x, x^{-1}\right]$. The next two propositions show that $P$ and $Q$ tell the whole story.

Proposition 2. A torsion injective graded $k[x]$-module is a direct sum of $k\left[x^{-1}\right]$ 's. The number of copies of $P_{m}$ is $\operatorname{dim}_{k}\left\{v \in V_{m}: x v=0\right\}$.

PROOF. If $\left\{y_{\lambda, i}\right\}_{\lambda=1}^{\alpha_{i}}$ is a basis for $V_{i}^{\prime}=\left\{v \in V_{i}: x v=0\right\}$, define $y_{\lambda, i}^{n}$ inductively for $n>0$ as any solution of $y_{\lambda, i}^{n-1}=x y_{\lambda, i}^{n}$. Then $\left\{y_{\lambda, i}^{\mu}\right\}_{\lambda, i, \mu \leq n}$ is linearly independent (induction on $n$ ) and spans $\left\{v \in V: x^{n} v=0\right\}$. So if $V$ is torsion,

$$
\begin{aligned}
V & =\bigcup_{n \geq 1}\left\{v \in V: x^{n} v=0\right\}=\bigoplus_{i, \lambda, \mu} k y_{\lambda, i}^{\mu} \\
& =\bigoplus_{\substack{i \\
1 \leq \lambda \leq \alpha_{i}}} k\left[x^{-1}\right] y_{\lambda, i} \cong \bigoplus_{i} k\left[x^{-1}\right]^{\left(\alpha_{i}\right)}
\end{aligned}
$$


Proposition 3. A torsion-free injective graded $k[x]$-module is a direct sum of copies of $Q=k\left[x, x^{-1}\right]$.

PROOF. If $\left\{y_{\lambda}\right\}$ is a basis of $V_{0}$, then $\left\{x^{i} y_{\lambda}\right\}$ is a basis of $V_{i}$. Hence $V \cong Q^{(\alpha)}$, where $\alpha=\operatorname{dim}_{k} V_{0}$.

All graded modules decompose as $R \oplus I$, where $R$ is reduced (i.e., contains no injective submodule) and $I$ is injective. It is this decomposition that allows us in the next section to accomplish the decomposition of (reduced) locally finite modules into the direct sum of cyclics.

III. The decomposition of reduced modules. A graded $k[x]$-module is bounded below if there is a lower bound on the degrees of the nonzero components. For example finitely generated modules have this property.

The simple half of the proof of Theorem 1 is split off as the following lemma. The lemma is of interest in itself.

LEMMA 1. If $V$ is torsion-free and bounded below, then $V$ is free.

Proof. For $i>0$, let $V_{i}=x V_{i-1} \oplus W_{i}$. Denote by $B_{i}$ a $k$-basis of $W_{i}$ and let $B_{0}$ be a $k$-basis of $V_{0}$. Then, assuming the nonzero components are bounded below by zero, $B=\bigcup_{i=0}^{\infty} B_{i}$ is a basis for $V$.

THEOREM 1. A graded $k[x]$-module $V$ that is bounded below is a direct sum of cyclic submodules.

Proof. If $T$ is the torsion submodule of $V$, then $V / T$ is free (Lemma 1 ). Therefore the problem reduces to the torsion case. In addition let us assume $V_{0}$ is the nonzero component of least degree.

Using Zorn's Lemma, choose a maximal linearly independent set $S_{0}$ of $V_{0}$ over $k[x]$.

The submodule $W$ generated by $S_{0}$ is pure in $V$. We claim that $W \cap V_{0}=V_{0}$. If not, and $v \in V_{0}-W$, let $\left(V_{0}\right)$ be the submodule generated by $V_{0}$ and $\bar{v} \in\left(V_{0}\right) / W$ the coset to which $v$ belongs. Suppose the order of $\bar{v}$ is $n$. Since $W$ is pure in $\left(V_{0}\right)$, there exists $v^{\prime} \in\left(V_{0}\right)$ such that $x v^{\prime}=0$ and $v^{\prime}-x^{n-1} v \in W[\mathbf{9}$, Lemma 1]. We can assume $v^{\prime}$ is homogeneous of degree $n-1$ and $v^{\prime}=x^{n-1} v^{\prime \prime}$ for some $v^{\prime \prime} \in V_{0}$. All of this means $S_{0} \cup\left\{v^{\prime \prime}\right\}$ is linearly independent, a contradiction that yields $W \cap V_{0}=V_{0}$.

Now suppose a linearly independent subset of $\bigcup_{i<n} V_{i}$ has been found that generates all homogeneous elements of degree $<n$. If $U$ is the submodule generated by these elements, then the least possibly nonzero component of $V / U$ is $V_{n} / V_{n} \cap U$.

Choose a subset $S_{n}^{\prime}$ of $V_{n} / V_{n} \cap U$ which is a maximal independent set, as in the previous part of this proof, and let $S_{n}$ be a subset of $V_{n}$ which is a set of coset representations for $S_{n}^{\prime}$. The union $\bigcup_{n>0} S_{n}$ is clearly linearly independent and generates $V$. Q.E.D.

As we shall describe after Theorem 2, the identity of countably generated graded $k[x]$-modules is completely known once we have ascertained the nature of the reduced torsion-free modules which are countably generated. First we need the concepts of height, local finiteness, and purity.

A graded $k[x]$-module is locally finite if every homogeneous component $V_{i}$ has finite $k$-dimension. The height $h(v)$ of an element $v \in V$ is the largest integer $n$ for 
which the equation $v=x^{n} w$ has a solution $w$. If no such $n$ exists, we say the height is infinite. A submodule $W$ of $V$ is pure if every equation $w=x^{n} z \in W$ which has a solution $z$ has a solution that lies in $W$.

LEMMA 2. If $V$ is locally finite the homogeneous elements of any given degree can assume only a finite number of different heights.

PROOF. In the homogeneous component $V_{i}$, the set of elements of height greater than or equal to $n$ (for any $n$ ) is a submodule. Since $V_{i}$ is finite dimensional, the descending chain condition implies that the set of heights is finite.

THEOREM 2. A countably generated reduced torsion-free $k[x]$-module $V$ is free.

Proof. Write $V$ as the union of an ascending chain $V_{1} \subseteq V_{2} \subseteq \cdots$ of pure submodules, where $V_{n}$ has finite rank. $V_{n+1} / V_{n}$ is reduced and locally finite. Therefore by Lemma 2 it is finitely generated and bounded below. Theorem 1 now implies $V_{n+1} / V_{n}$ is a direct sum of cyclics. So by $[\mathbf{9}$, Theorem 5], whose proof carries over to the graded case, $V_{n}$ is a direct summand of $V_{n+1}$. Using induction and assuming $V_{n}$ is free, this implies $V_{n+1}$ is also free. Hence $V$ itself is free. Q.E.D.

Theorem 2 tells us that, in general, a countably generated graded $k[x]$-module is the direct sum of its reduced torsion submodule, a free module, and an injective module. The nature of countably generated reduced torsion modules is described by Ulm's Theorem [9] whose proof carries over to the graded case. Therefore the study of countably generated modules is complete.

Although countably generated torsion-free modules are free (Theorem 2), it is certainly not true that uncountably generated torsion-free modules are free. The following example illustrates this.

EXAMPLE. For integers $m$, let $k_{m}$ be a copy of the field $k$. Define $V_{i}$ to be $\prod_{m \geq 0} k_{m}$ if $i \geq 0$ and $\prod_{m \geq-i} k_{m}$ if $i<0$. Grade the $V_{i}$ 's by $x_{i}: V_{i} \rightarrow V_{i+1}$ which is the identity if $i \geq 0$ and the canonical injection if $i<0$. To be free, it is seen easily enough, $V$ would have to have a countable basis as a $k$-space. This is manifestly not true.

LEMMA 3. If $V$ is reduced and locally finite, then $V$ has no nonzero elements of infinite height.

Proof. Suppose $0 \neq v \in V_{i}$ and $h(v)=\infty$. By Lemma 2, there must exist $w_{i} \in$ $V_{i-1}$ with $v=x w_{1}$ and $h\left(w_{1}\right)=\infty$. So we have a sequence $v=x w_{1}=x^{2} w_{2}=\cdots$. This means $V$ contains a copy of $k\left[x^{-1}\right]$ or $k\left[x, x^{-1}\right]$, depending on whether $v$ is a torsion element or not. This contradiction establishes the lemma.

THEOREM 3. A reduced and locally finite graded $k[x]$-module is a direct sum of cyclic submodules.

ProOF. If $T$ is the torsion submodule of $V$, then $V / T$ is countably generated. $V / T$ is also reduced for if $\bar{v}=x^{m} \bar{w}$, then $x^{m} w=v+t$ for $t \in T$ and we may assume $w, v$ and $t$ are homogeneous. So $m$ must be less than or equal to the maximum (finite) height in the homogeneous component containing $v$ and $t$. Thus $\bar{v}$ cannot have infinite height and $V / T$ is consequently reduced. Theorem 2 now implies $V / T$ is free. We may assume, therefore, $V$ is torsion. But in this case, Lemma 3 guarantees $V$ contains no elements of infinite height. Prüfer's theorem [9, Theorem 11] is that a countably generated torsion module with no elements of 
infinite height is a direct sum of cyclic submodules. (The proof of the ungraded version in [9] carries over to the graded case.) Therefore an application of Prüfer's theorem completes the proof.

REMARK. The proof actually suffices to prove the bit stronger statement wherein not all components of the torsion submodule need be finite dimensional, but only those whose degree is less than a certain upper bound.

IV. Pure submodules and direct summands. Torsion submodules and direct summands are always pure. Other elementary properties of purity are that it is transitive and that the union of a chain of pure submodules is pure.

An immediate corollary of Theorem 2 is that a pure submodule of a countably generated torsion-free module is necessarily a direct summand.

In this section it is shown how pure submodules may in fact be direct summands. Theorem 4 will tell us it is only necessary they be bounded below. Along the way, Proposition 4 will show that uncountably generated reduced torsion-free modules have free summands of countable rank. (Theorem 2 proved countably generated reduced torsion-free modules are free.)

PROPOSITION 4. An uncountably generated reduced torsion-free graded $k[x]$ module has free summands whose ranks exceed any integer.

ProOF. Fix an integer $m$. Write $V_{m}=x V_{m-1} \oplus W_{m}$. Then we can write

$$
V_{m+1}=x^{2} V_{m-1} \oplus x W_{m} \oplus W_{m}^{\prime}=x^{2} V_{m-1} \oplus W_{m+1},
$$

where $W_{m+1}=x W_{m}+W_{m}^{\prime}$. By induction,

$$
V_{i}=x^{i-m+1} V_{m-1} \oplus W_{i} \quad \text { for } i>m,
$$

where the submodule $W$ generated by the $W_{i}$ 's, $i \geq m$, is a direct summand that is bounded below. By Lemma 1 (or Theorem 1) $W$ is free. Since $V$ is reduced $m$ can be chosen so that the rank of $W$ exceeds any integer.

LEMMA 4. Suppose $V$ is torsion-free and $W$ is a pure submodule that is bounded below. Then $W$ is a direct summand.

Proof. Let us assume the nonzero homogeneous components of $W$ are of nonnegative degree. Note that $W_{0} \cap x V_{-1}=0$ because $W$ is pure. So we may write $V_{0}=W_{0} \oplus U_{0}$, where $U_{0}$ can be chosen to contain $x V_{-1}$.

Define $U_{i}=V_{i}$ for $i<0$ and assume $U_{0}, \ldots, U_{m}$ have been chosen so that $V_{i}=W_{i} \oplus U_{i}$ and $x U_{i-1} \subset U_{i}$ for $i=-1, \ldots, m$. Note that $W_{m+1} \cap x U_{m}=0$ since $W$ is pure and $W_{m} \cap U_{m}=0$. (It is here the hypothesis of torsion-freeness is used.) So we may write $V_{m+1}=W_{m+1} \oplus U_{m+1}$, where $U_{m+1}$ contains $x U_{m}$. By induction, therefore, $V_{i}=W_{i} \oplus U_{i}$, for all $i$, where $U=\bigoplus U_{i}$ is a complement of $W$ in $V$.

REMARK. If in the Example of a nonfree reduced torsion-free module, $W$ is the submodule whose $i$ th component is the direct sum of the $k_{m}$ 's, then $W$ is pure and $V / W$ is injective. Were $W$ a summand, $V$ would then necessarily contain a nonzero injective submodule. This shows that the condition of boundedness in the lemma and later in Theorem 4 is essential. 
LEMMA 5. If $V$ is torsion, bounded below, and $W$ is a pure submodule, then $W$ is a direct summand.

PROOF. If $V_{0}$ is the nonzero component of least degree in $V$, then it suffices to suppose $W_{0}$ is the nonzero component of least degree in $W$. (This small point is justified in the proof of Theorem 4.) The proof is now just a variation of the proof of Theorem 1. The two minor changes are to choose (1) $S_{0}=R_{0} \cup T_{0}$, where $R_{0}$ generates $W_{0}$, and (2) partition $S_{n}$ into $R_{n} \cup T_{n}$, where $R_{n}$ generates the complement in $W_{n}$ of $U$.

THEOREM 4. If $W$ is bounded below and is a pure submodule of a graded $k[x]-$ module $V$, then $W$ is a summand of $V$.

PROOF. Assume without loss of generality that $W_{0}$ is the nonzero component of $W$ of least degree. Let $U$ be the submodule of $V$ generated by the homogeneous components $V_{i}, i<0$. Because $W$ is pure and $U \cap W=0$, the canonical function embeds $W$ as a pure submodule of $V / U$.

If $W_{T}$ and $T$ are the torsion submodules of $W$ and $V / U$ respectively, then by Lemma 1 torsion-free complements $W_{F}$ and $F$ can be chosen so that $W=W_{T} \oplus W_{F}$, $V / U=T \oplus F, W_{T} \subseteq T$ and $W_{F} \subseteq F$. That $W_{T}$ is a summand of $T$ follows from Lemma 5. That $W_{F}$ is a summand of $F$ follows from Lemma 4. Thus $W$ embeds as a summand of $V / U$, consequently $W$ is also a summand of $V$.

REMARK. Theorems 1 and 4 can be unified in the following way. In doing so we make use of the convention that a submodule may be improper, in other words, be the whole module.

THEOREM 5. If a pure submodule of a graded $k[x]$-module is bounded below, then it is a direct summand and is itself a direct sum of cyclic submodules.

V. Stacked Bases Theorem. In 1969 Cohen and Gluck proved the so-called Stacked Bases Theorem for modules over a PID [1, 2]. This theorem asserts that the quotient of two free modules is a direct sum of cyclic modules if and only if there is a basis of the contained module whose elements are multiples of elements in a subset of some basis of the containing module.

In proving the graded version of this theorem there are, in addition to Cohen and Gluck's proof of the ungraded version, only two minor points that might be made. (1) When selecting a basis of the containing graded module, choose a homogeneous one. (2) The graded finite dimensional theorem is true. (The proof, cited by Cohen and Gluck in $[\mathbf{1 0}]$, goes through verbatim.)

\section{REFERENCES}

1. J. Cohen and H. Gluck, Stacked bases for modules, Bull. Amer. Math. Soc. 75 (1969), 978-979.

2. _ Stacked bases for modules, J. Algebra 14 (1970), 493-505.

3. S. Eilenberg, Homological dimension and syzygies, Ann. of Math. (2) 64 (1956), 328-336.

4. P. Gabriel, Representations indecomposables des ensembles ordonnes, Seminaire P. Dubreil, 26e anee (1972/73), No. 13, Secretariat Mathematique, Paris, 1973.

5. __ Problemes actuels de theorie des representations, Enseign. Math. (2) 20 (1974), 323-332.

6. __ Representations indecomposables (Seminaire Bourbaki, 26e anee (1973/74), No. 444, pp. 143-169), Lecture Notes in Math., vol. 431, Springer, Berlin, 1975.

7. P. Gabriel and C. Riedtmann, Group representations without groups, Comment. Math. Helv. 54 (1979), 240-287. 
8. M. Höppner and H. Lenzing, Diagrams over ordered sets: a simple model of abelian group theory, Abelian Group Theory, Proceedings Oberwolfach 1981 (R. Göbel and E. Walker, eds.), Lecture Notes in Math., vol. 874, Springer-Verlag, Berlin, 1981.

9. I. Kaplansky, Infinite abelian groups, (rev. ed.), Univ. of Michigan Press, Ann Arbor, 1969.

10. C. Sah, Abstract algebra, Academic Press, New York, 1967.

11. C. Webb, Graded modules bounded below are direct sums of cyclics, Notices Amer. Math. Soc. 22 (1975), A-400.

Department of Mathematics and Computer Science, Chicago State UniverSITY, CHICAGO, ILliNOIS 60628 